International Journal of Engineering \& Technology, $7(2.24)(2018) 172-176$
International Journal of Engineering \& Technology
SPC
Website: www.sciencepubco.com/index.php/IJET
Research paper

\title{
The Quadruple Tank Process with an Interaction : A Mathematical Model
}

\author{
E.Govinda Kumar1*, B .Shiva ram $^{2}$, U.B.Deepak ${ }^{3}$, G.Sabarinathan ${ }^{4}$ \\ ${ }^{1}$ Assistant Professor, Department of Electronics and Instrumentation Engineering, Karpagam College of Engineering, Coimbatore \\ ${ }^{2,3,4}$ Department of Electronics and Instrumentation Engineering, Karpagam College of Engineering, Coimbatore \\ *Corresponding Author Email: egovindakumar@gmail.com
}

\begin{abstract}
This paper dealt that, the quadruple tanks process with an interaction, which is consisting of four interconnected tanks and included with an interaction of bottom two tanks. The mathematical model of quadruple tankwith interaction is developed throughminimum phase and non-minimum phase by changing a valve position. It described clearly about the mathematical modeling of quadruple-tanks process with an interaction, which is obtained based on minimum and non-minimum characteristics. The obtained process transfer function models are validated using MATLAB simulation. The obtained transfer function models are used to further control and analysis of the same problem.
\end{abstract}

Keywords: Quadruple Tank Process; Interacting Process; Process Modeling;Transfer Function Matrix; MIMO and TITO process.

\section{Introduction}

Most of the industrial processes are multi-input and multi-output (MIMO) environment [1] and it has strongly integrated process, which shows complex dynamics and nonlinear behavior [2]. These are common for industrial processes and it has significant uncertainties, strong interaction of minimum and non-minimum phase behavior. A great attention is received for the control of multivariable quadruple tank process (QTP) and it is motivatingthe both control learning and research [4]. The characteristics of multivariable QTP are investigated by using the values of two flow ratio with a same experimental apparatus [5]. It is suited for the purpose of analyses and illustrate various designs in multivariable control, mostly performance limitation due to non-minimum-phase zero [6].

The developments of process models are play a more important role for the control of process and analysis of process behavior. In process industries the process modeling are complicated for of MIMO process. It has more than one manipulated variable and more than one control input, and the interactions between these are increase the complexity for the development of process models. In this work simulation and mathematical models of Quadruple Tank Process with Interaction (QTPI) has been discussed. In chemical engineering, the transfer function matrices are frequently used for the analyses and control applications [7] The number of inputs in the MIMO process is greater than/equal to number of outputs, and then this MIMO process is controllable. The QTP contains the equal number of inputs and outputs are considered for the development of

Mathematical model. This work aims to achieve the process transfer function matrices for minimum and non-minimum phase characteristics of QTPI process.

This paper is structured as follows. Section two describes the quadruple tank process with interaction. Section three is about the development of mathematical modeling of QTPI process. Simulation results are given in Section four. Conclusions are drawn in Section five.

\section{Description of Quadruple Tank Process with Interaction}

The bench mark QTP has consists four cylindrical tanks which are placed vertically with two different location and these are arranged as shown in Fig.1 [1, 3, 8, 9]. The area of tank 1 and tank 3 is $28 \mathrm{~cm}^{2}$ and their outlet pipes are connected to outlet valve with flow resistance for outlet flowing. The area of tank 2 and tank 4 is $30 \mathrm{~cm}^{2}$ and their outlet pipes are connected to outlet valve with flow resistance for outlet flowing. The store water for the process using the reservoir tank and the water is flows in all the four tanks two pumps are used.

The QTP process contains the four water tanks and two pumps. The aim is to control the water level in the lower tank 1 and 2 with the two pumps. The inputs of the QTP process are the input voltages of the pumps $\left(V_{1}, V_{2}\right)$ and the outputs are the liquid levels in the lower tanks $\left(h_{1}, h_{2}\right)$. The flow from pump is split up in a part proportional to $\gamma_{i}$ and a part proportional to $1-\gamma_{i}$. The flows of the pumps are split up by valves. 


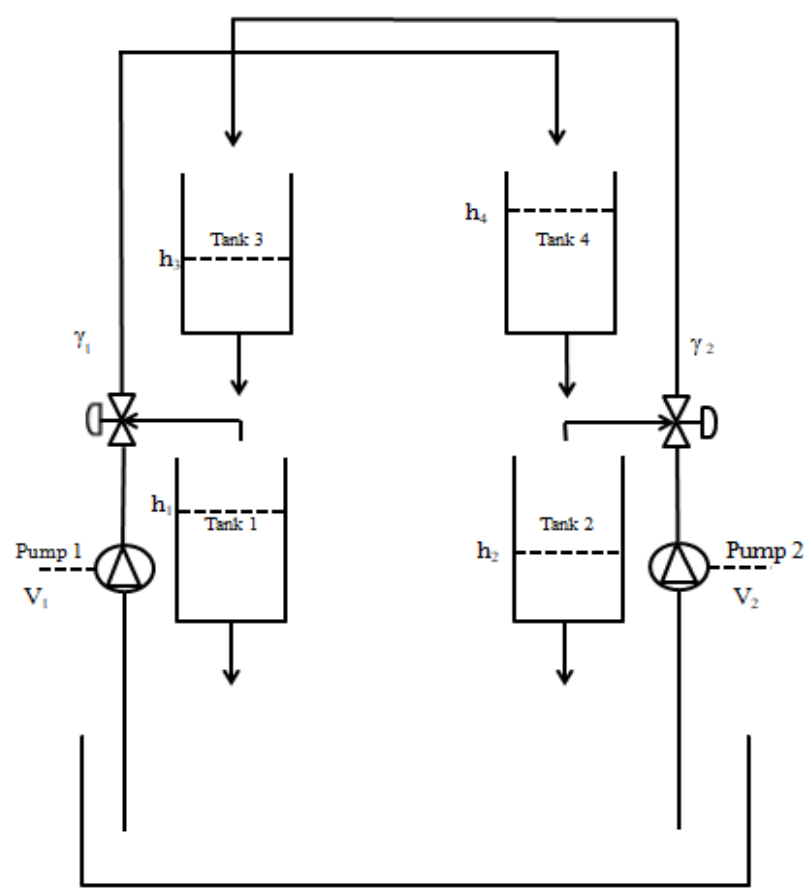

Fig. 1: The quadruple tank process

The physical model of the quadruple-tank with an interaction is developed from the modification of bench mark quadruple tank process (QTP). The modification of existing QTP is creating the interaction between tank 1 and tank 2 . A hole is bored at the bottom tank 1 and tank 2 to the water from each tank to flow with the pipe is connected between the tanks. Fig. 2 shows that the quadruple-tank process with an interaction (QTPI).

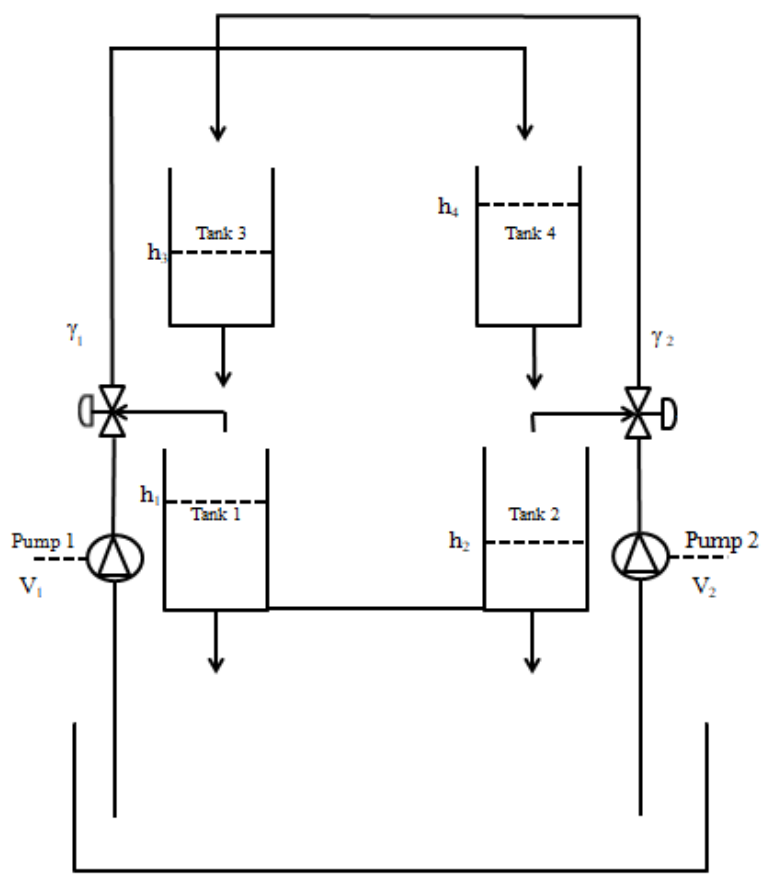

Fig.2: The quadruple tank process with an interaction

A one input and it is split the two outlet flows which are directed to a bottom and upper level tanks respectively. The Pump 1 and pump 2 flow rates are directly connected to the bottom two tanks. The QTPI process can be operated with the transition zeros is in the right half plane and left half plane by adjusting the valve knob.
The each tank of QTPI process consists of one or more than one input flow and output flow. The tank volume is proportional to area of the tank and actual level inside the tank. The parameter $h(t)$ is a time varying with input and output flow changes. The mass balance equations of QTPI process are given below equation. The following state equations are represents the nonlinear model of the QTPI process,

$$
\begin{aligned}
& \frac{d h_{1}(t)}{d t}=-\frac{a_{1}}{A_{1}} \sqrt{2 g h_{1}(t)}+\frac{a_{3}}{A_{1}} \sqrt{2 g h_{3}(t)}-\frac{a_{12}}{A_{1}} \sqrt{2 g\left|h_{1}(t)-h_{2}(t)\right|}+\frac{\gamma_{1} K_{1}}{A_{1}} V_{1} \\
& \frac{d h_{2}(t)}{d t}=-\frac{a_{2}}{A_{2}} \sqrt{2 g h_{2}(t)}+\frac{a_{4}}{A_{2}} \sqrt{2 g h_{4}(t)}-\frac{a_{12}}{A_{1}} \sqrt{2 g\left|h_{1}(t)-h_{2}(t)\right|}+\frac{\gamma_{2} K_{2}}{A_{2}} V_{2} \\
& \frac{d h_{3}(t)}{d t}=-\frac{a_{3}}{A_{3}} \sqrt{2 g h_{3}(t)}+\frac{\left(1-\gamma_{2}\right) K_{2}}{A_{3}} V_{2} \\
& \frac{d h_{4}(t)}{d t}=-\frac{a_{4}}{A_{4}} \sqrt{2 g h_{4}(t)}+\frac{\left(1-\gamma_{1}\right) K_{1}}{A_{4}} V_{1}
\end{aligned}
$$

The process parameters of QTPI process are given in Table 1 . These parameters are used to develop simulation model and transfer function matrices of QTPI process.

Table. 1: QTPI process parameters

\begin{tabular}{c|c} 
Table. 1: QTPI process parameters \\
\hline Parameters(unit) & Value \\
\hline$A_{1}, A_{3}\left[\mathrm{~cm}^{2}\right]$ & 28 \\
\hline$A_{2}, A_{4}\left[\mathrm{~cm}^{2}\right]$ & 32 \\
\hline$a_{1}, a_{2}\left[\mathrm{~cm}^{2}\right]$ & 0.071 \\
\hline$a_{3}, a_{4}\left[\mathrm{~cm}^{2}\right]$ & 0.057 \\
\hline$K_{c}\left[V / \mathrm{cm}^{2}\right]$ & 1 \\
\hline$g\left[\mathrm{~cm}^{2} \mathrm{~s}^{2}\right]$ & 981 \\
\hline$k_{1}\left[\mathrm{~cm}^{3} / \mathrm{Vs}\right]$ & 3.14 \\
\hline$k_{2}\left[\mathrm{~cm}^{3} / \mathrm{Vs}\right]$ & 3.29 \\
\hline$\gamma_{1}$ & $0.35,0.6$ \\
\hline$\gamma_{2}$ & $0.35,0.6$ \\
\hline
\end{tabular}

\section{Modeling of quadruple tank process with interaction}

This section aims at description of process models and develops mathematical models of QTPI process. The mathematical models of QTPI process is developed through minimum and nonminimum phase characteristics.

The nonlinear model of QTPI process is linearized with the operation point is given by given by the liquid height of the tanks $h_{1}^{0}, h_{2}^{0}, h_{3}^{0}$ and $h_{4}^{0}$. The above nonlinear differential equations are converted to linearized state space model by using Jacobian Matrix. The Jacobian matrix is represented as,

$$
\frac{\partial f}{\partial h}=\left[\begin{array}{llll}
\frac{\partial f_{1}}{\partial h_{1}} & \frac{\partial f_{1}}{\partial h_{2}} & \frac{\partial f_{1}}{\partial h_{3}} & \frac{\partial f_{1}}{\partial h_{4}} \\
\frac{\partial f_{2}}{\partial h_{1}} & \frac{\partial f_{2}}{\partial h_{2}} & \frac{\partial f_{2}}{\partial h_{3}} & \frac{\partial f_{2}}{\partial h_{4}} \\
\frac{\partial f_{3}}{\partial h_{1}} & \frac{\partial f_{3}}{\partial h_{2}} & \frac{\partial f_{3}}{\partial h_{3}} & \frac{\partial f_{3}}{\partial h_{4}} \\
\frac{\partial f_{4}}{\partial h_{1}} & \frac{\partial f_{4}}{\partial h_{2}} & \frac{\partial f_{4}}{\partial h_{3}} & \frac{\partial f_{4}}{\partial h_{4}}
\end{array}\right]
$$

Using the Eqn. (2), the linearized state space model for QTPI process as, 


$$
\begin{aligned}
& \dot{x}=A x+B u \\
& y=C x
\end{aligned}
$$

Using Jacobian matrix, determine the state space model of the QTPI process is represented as,

$A=\left[\begin{array}{cccc}-\frac{1}{T_{1}}-\frac{1}{T_{12}} & \frac{1}{T_{12}} & \frac{A_{3}}{A_{1} T_{3}} & 0 \\ \frac{1}{T_{12}} & -\frac{1}{T_{2}}-\frac{1}{T_{21}} & 0 & \frac{A_{3}}{A_{1} T_{3}} \\ 0 & 0 & -\frac{1}{T_{3}} & 0 \\ 0 & 0 & 0 & -\frac{1}{T_{4}}\end{array}\right]$

$B=\left[\begin{array}{cc}\frac{\gamma_{1} K_{1}}{A_{1}} & 0 \\ 0 & \frac{\gamma_{2} K_{2}}{A_{2}} \\ 0 & \frac{\left(1-\gamma_{2}\right) K_{2}}{A_{3}} \\ \frac{\left(1-\gamma_{1}\right) K_{1}}{A_{4}} & 0\end{array}\right]$

$C=\left[\begin{array}{cccc}K_{c} & 0 & 0 & 0 \\ 0 & K_{C} & 0 & 0\end{array}\right]$

From the above state space model, the transfer function matrix of QTPI process is determined using the below equation.

$G(s)=C(S I-A)^{-1} B+D$

The process transfer function matrix General TITO process represents as,

$$
G(s)=\left[\begin{array}{ll}
G_{11}(s) & G_{12}(s) \\
G_{21}(s) & G_{22}(s)
\end{array}\right]
$$

The following matrix is represents the process transfer function matrix of QTPI process.

$G(s)=\left[\begin{array}{cc}\frac{K_{1} \gamma_{1}\left(S+\frac{1}{T_{2}}+\frac{1}{T_{21}}\right)\left(S+\frac{1}{T_{4}}\right)-\frac{K_{24}}{T_{12}}\left(1-\gamma_{1}\right)}{\left(S+\frac{1}{T_{4}}\right) x(s)} & \frac{\frac{K_{2}}{T_{12}} \gamma_{2}\left(S+\frac{1}{T_{3}}\right)+K_{13}\left(1-\gamma_{2}\right)\left(S+\frac{1}{T_{2}}+\frac{1}{T_{21}}\right)}{\left(S+\frac{1}{T_{3}}\right) x(s)} \\ \frac{K_{1}}{T_{21}} \gamma_{1}\left(S+\frac{1}{T_{4}}\right)+K_{24}\left(1-\gamma_{1}\right)\left(S+\frac{1}{T_{1}}+\frac{1}{T_{12}}\right) \\ \left(S+\frac{1}{T_{4}}\right) x(s) & \frac{K_{22} \gamma_{2}\left(S+\frac{1}{T_{1}}+\frac{1}{T_{12}}\right)\left(S+\frac{1}{T_{3}}\right)-\frac{K_{12}}{T_{21}}\left(1-\gamma_{2}\right)}{\left(S+\frac{1}{T_{3}}\right) x(s)}\end{array}\right]$

Where,

$x(s)=\left(S+\frac{1}{T_{1}}+\frac{1}{T_{12}}\right)\left(S+\frac{1}{T_{2}}+\frac{1}{T_{21}}\right)-\frac{1}{T_{12}} \cdot \frac{1}{T_{21}}$

$K_{i}=\frac{K_{c} k_{i}}{A_{i}}$ for $i=1,2$

$T_{i}=\frac{A_{i}}{a_{i}} \sqrt{\frac{2 h_{i}}{g}}$ for $\mathrm{i}=1$ to 4
$T_{12}=\frac{A_{1}}{a_{1}} \sqrt{\frac{2\left(h_{1}-h_{2}\right)}{g}}$

$T_{21}=\frac{A_{2}}{a_{2}} \sqrt{\frac{2\left(h_{1}-h_{2}\right)}{g}}$

$K_{13}=\frac{K_{c} k_{2}}{A_{1} T_{3}}$

$K_{24}=\frac{K_{c} k_{1}}{A_{2} T_{4}}$

The QTPI process transfer function matrixes are obtained with the input and output operating points are given in Table 2.

Table 2: Operating points of QTPI process

\begin{tabular}{c|c}
\hline Parameters(unit) & Value \\
\hline$h_{1}^{0}[\mathrm{~cm}]$ & 10.43 \\
\hline$h_{2}^{0}[\mathrm{~cm}]$ & 15.98 \\
\hline$h_{3}^{0}[\mathrm{~cm}]$ & 6.60 \\
\hline$h_{4}^{0}[\mathrm{~cm}]$ & 9.57 \\
\hline$v_{1}^{0}[\mathrm{~V}]$ & 3.15 \\
\hline$v_{2}^{0}[\mathrm{~V}]$ & 3.15 \\
\hline
\end{tabular}

The mathematical model of non-minimum characteristics QTPI process is obtained by choosing the values $\gamma_{1}, \gamma_{2}$ are to be satisfy $0 \leq \gamma_{1}+\gamma_{2} \leq 1$. The values of $\gamma_{1}, \gamma_{2}$ are chosen as 0.35 and the process transfer function matrix is given in Eqn. 17.

$G(s)=\left[\begin{array}{cc}\frac{0.0392 S^{2}+0.0017 S+0.00001}{S^{3}+0.0968 S^{2}+0.01 S+0.0001} & \frac{0.0026 S+0.00007}{S^{3}+0.1066 S^{2}+0.0108 S+0.0001} \\ \frac{0.0014 S+0.00004}{S^{3}+0.0968 S^{2}+0.018 S+0.0001} & \frac{0.0357 S^{2}+0.002 S+0.00003}{S^{3}+0.1066 S^{2}+0.0108 S+0.0001}\end{array}\right]$

The mathematical model of minimum characteristics QTPI process is obtained by choosing the values $\gamma_{1}, \gamma_{2}$ are to be satisfy $1 \leq \gamma_{1}+\gamma_{2} \leq 2$. The values of $\gamma_{1}, \gamma_{2}$ are chosen as 0.6 and the process transfer function matrix is given in Eqn. 18.

$G(s)=\left[\begin{array}{cc}\frac{0.0672 S^{2}+0.003 S+0.00001}{S^{3}+0.0968 S^{2}+0.01 S+0.0001} & \frac{0.0028 S+0.00006}{S^{3}+0.1066 S^{2}+0.0108 S+0.0001} \\ \frac{0.0016 S+0.00003}{S^{3}+0.0968 S^{2}+0.01 S+0.0001} & \frac{0.0612 S^{2}+0.004 S+0.0009}{S^{3}+0.1066 S^{2}+0.0108 S+0.0001}\end{array}\right]$

\section{Simulation Results}

The rate of change of liquid level and it relates the difference between inlet and outlet flows. These relations of all the tanks are represented in Eqn.1. Using these equations, the simulation model of QTPI is developed and the values of process parameters are given in Table 1. The simulated responses of tank level height of the bottom tank 1 and tank 2 is given in Fig. 3 and Fig. 4

The values of flow coefficients $\gamma_{1}$ and $\gamma_{2}$ are considered as 0.3 and 0.3 respectively. The summation of these two values are belongs to $0 \leq \gamma_{1}+\gamma_{2} \leq 1$, which is minimum phase characteristics of QTPI process. The open loop responses of minimum phase characteristics of QTPI process is shown inFig. 3. 


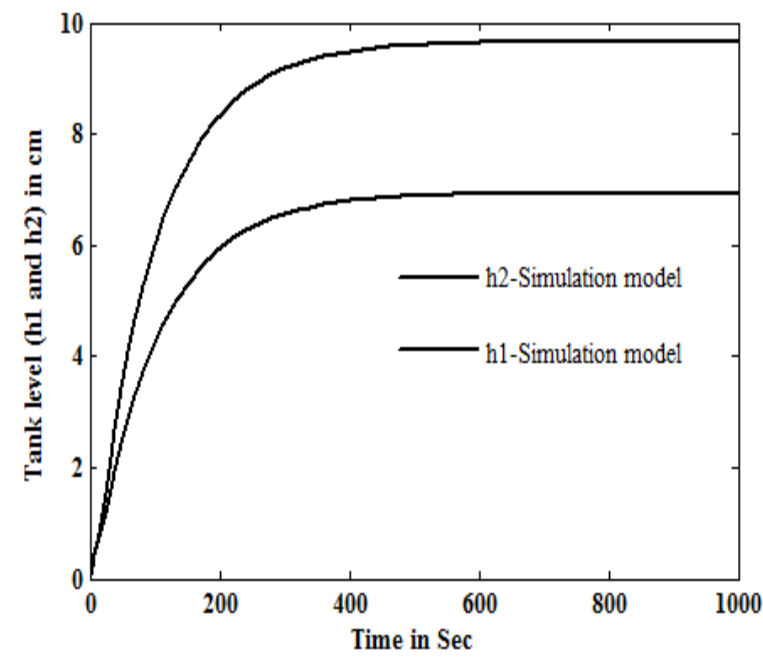

Fig. 3: Open loop responses of simulation model of QTPI with minimum phase characteristics

The range of flow coefficients $\gamma_{1}$ and $\gamma_{2}$ are preferred to $1 \leq \gamma_{1}+\gamma_{2} \leq 2$, which is belongs to non-minimum phase characteristics of QTPI process. The values $\gamma_{1}$ and $\gamma_{2}$ are considered as 0.3 and 0.3 respectively. The open loop responses of non-minimum phase characteristics of QTPI process is shown in Fig. 4.

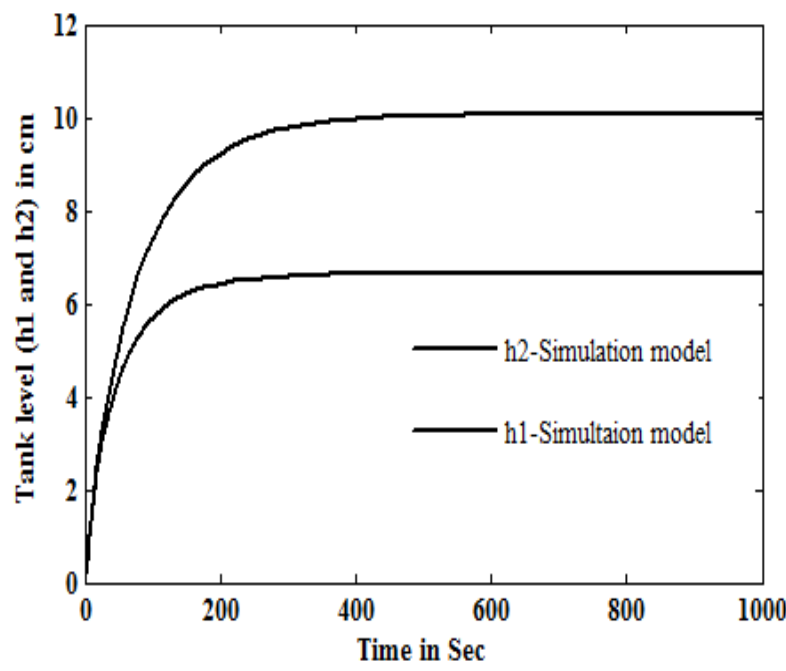

Fig. 4: Open loop responses of simulation model of QTPI with minimum phase characteristics

From Table 1 and Table 2, these process parameters are used to develop a mathematical model of QTPI process. The mathematical model of non-minimum phase characteristics of QTPI process is given in Eqn. 17. The values of flow coefficients are changed to develop a mathematical model of minimum characteristics of QTPI process and it is given in Eqn. 18.

The above obtained process models are validated by using Matlab simulation. The open loop responses of obtained mathematical models are compared with the simulated responses of QTPI process. The comparison of the open loop responses are given in Fig. The responses of simulation and mathematical model of nonminimum characteristics of QTPI process are shown in Fig. 5.

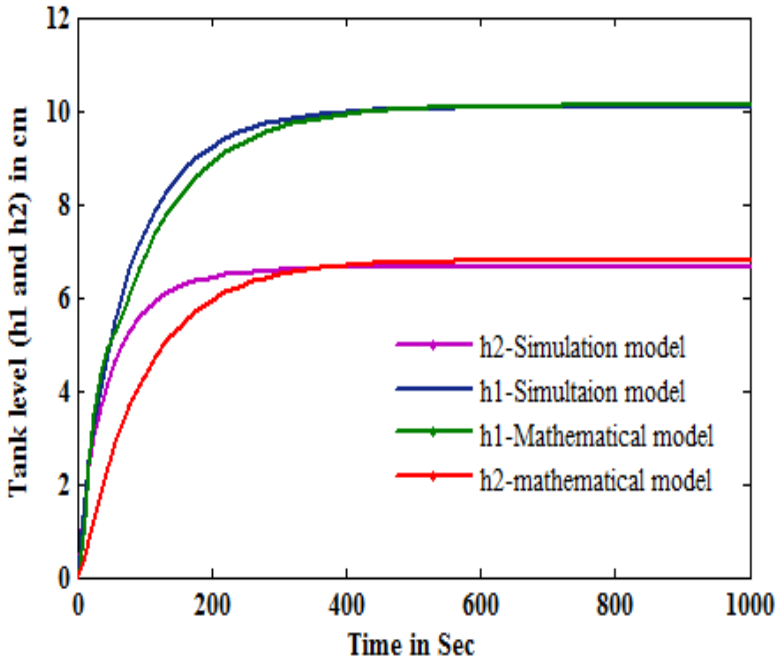

Fig. 5: Open loop responses of QTPI with non-minimum phase characteristics

Fig. 6 shows that the comparison of the open loop responses of simulation and mathematical models of minimum phase characteristics of QTPI process.

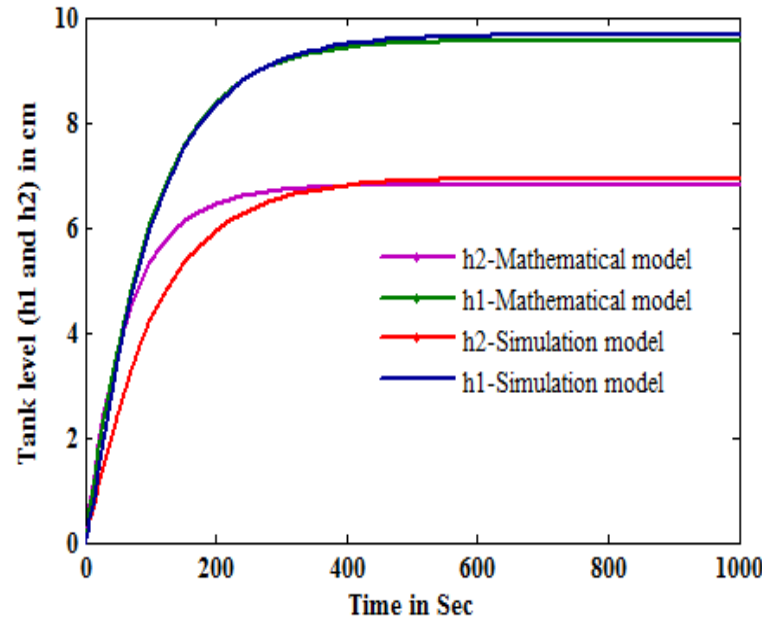

Fig.6: Open loop responses of QTPI with minimum phase characteristics

From Fig. 5 and Fig. 6, it can be seen that the responses simulation and mathematical models are identical. The responses of non-minimum and minimum characteristics of QTPI process are same in steady state values of tank 1 . The responses of nonminimum and minimum characteristics of QTPI process are very close steady state values of tank 2 . From the results, it can be observed that the obtained mathematical model of non-minimum and minimum phase characteristics response of QTPI is the same as simulated models.

\section{Conclusion}

In this work, mathematical models of minimum and non-minimum phase characteristicsof QTPI process are developed. The simulated model of QTPI process is constructed and open loop responses of bottom tank 1 and tank 2 are obtained for minimum and non-minimum phase characteristics. The responses of obtained mathematical model are also obtained and it is compared with the responses of simulated model. From the comparison, it can be seen that the both responses are almost identical. Hence it is claimed that obtained process transfer functions with minimum and non-minimum phase characteristics of QTPI process are used 
to analyses the process behavior. It is also used for development of control law for the control of bottom two tanks.

\section{References}

[1] Rajapandiyan, C., and Chidambaram.M "Controller design for MIMO processes based on simple decoupled equivalent transfer functions and simplified decoupler." Industrial \& Engineering Chemistry Research Vol. 51, No. 38 (2012), pp.12398-12410.

[2] GovindaKumar E, Mithunchakravarthi B, and Dhivya N. "Enhancement of PID controller performance for a quadruple tank process with minimum and non-minimum phase behaviors." Procedia Technology vol. 14, (2014), pp.480-489.

[3] Johansson, K H, "The quadruple-tank process: A multivariable laboratory process with an adjustable zero," IEEE Transactions on control systems technology Vol.8, No. 3 (2000), pp. 456-465.

[4] Dormido, Sebastián, and Francisco Esquembre. "The quadrupletank process: An interactive tool for control education." In European Control Conference (ECC), 2003, pp. 3267-3272. IEEE, 2003.

[5] Roinila, Tomi, MattiVilkko, and AnttiJaatinen. "Corrected mathematical model of quadruple tank process." IFAC Proceedings Volumes Vol. 41, No. 2 (2008), pp.11678-11683.

[6] Shneiderman, D., and Palmor.Z. J. "Properties and control of the quadruple-tank process with multivariable dead-times." Journal of Process Control Vol. 20, No. 1 (2010), pp. 18-28.

[7] Corriou, Jean-Pierre. "Multivariable Control by Transfer Function Matrix." In Process Control, pp. 305-338. Springer, Cham, 2018.

[8] Biswas, PinakPani, Rishi Srivastava, Subhabrata Ray, and Amar NathSamanta. "Sliding mode control of quadruple tank process." Mechatronics Vol. 19, No. 4 (2009), pp.548-561.

[9] Kirubakaran, V., RadhakrishnanT. K., and. Sivakumaran N. "Distributed multiparametric model predictive control design for a quadruple tank process." Measurement Vol. 47 (2014),pp. 841854. 